\title{
Quality of life amongst lymphoma survivors in a developing country
}

\author{
Diana L. C. $\mathrm{Ng}^{1}$ • Y. C. Leong ${ }^{2}$ - Gin Gin Gan ${ }^{2}$
}

Received: 25 January 2016 / Accepted: 18 July 2016

(C) Springer-Verlag Berlin Heidelberg 2016

\begin{abstract}
Purpose The survival for patients with lymphoma has improved over the past decades with the introduction of novel agents. Quality of life of these survivors is now being studied with focus on minimising the late effects of chemotherapy and improving psychosocial support. This study aims to determine the prevalence of anxiety and depression of lymphoma survivors and to investigate the possible association between these disorders and quality of life.

Methods Patients with previous diagnosis of lymphoma who remained in remission were recruited from a major hospital in Malaysia. Quality of life of these patients was measured using European Organisation for Research and Treatment of Cancer Quality of Life Questionnaire (EORTC QLQ C30). Anxiety and depression symptoms were assessed using Hospital Anxiety and Depression scale (HADS).

Results A total of 156 patients participated in this study. Eighteen percent (18\%) of patients had symptoms of anxiety, and $10 \%$ had symptoms of depression. Patients who had higher depression scores were older, of lower education level and had more than one comorbidity illness. Patients with anxiety were associated with lower overall quality of life (QOL) score, lower emotional and cognitive functioning and complained more of fatigue and insomnia $(p<0.05)$. Patients who had depression were associated with lower physical functioning and complained more of insomnia $(p<0.05)$.
\end{abstract}

Gin Gin Gan

gangg@ummc.edu.my

1 Faculty of Medicine and Health Sciences, Universiti Malaysia Sarawak, Kota Samarahan, Malaysia

2 Department of Medicine, Faculty of Medicine, Universiti Malaya, Kuala Lumpur, Malaysia
Conclusion It is important for the treating physicians to ensure follow-up of lymphoma survivors for any psychological disorders in order early counseling and support can be provided. This may improve patients' quality of life.

Keywords Anxiety $\cdot$ Depression $\cdot$ Lymphoma $\cdot$ Survivors · Quality of life

\section{Introduction}

Lymphoid neoplasms are one of the most common haematological cancers. In the USA, $84 \%$ of haematological cancers are lymphoid neoplasms [1]. Non-Hodgkin lymphoma (NHL) accounted for approximately $80 \%$ of all lymphoid neoplasms, and Hodgkin lymphoma (HL) accounted for $8 \%$ [2]. NHL is the eighth most common cancer in the USA, and in Malaysia, lymphoma is the sixth most common cancer accounting for about $4.3 \%$ of all cancers $[3,4]$. Survival had improved significantly for both NHL and HL in the past decades in many countries including Asian regions [4-6]. The much improved survival rates and curability for lymphoma in recent years are the result of introduction of monoclonal antibody therapy in combination of chemotherapy drugs and newer radiotherapy technology [7-9]. In Malaysia, a recent study showed the 3year overall survival rate of patients with DLBCL was $80 \%$ [10]. With improved survival, patients are now reporting more treatment-related side effects such as cardiac dysfunction, secondary malignancies and gonadal dysfunction. There have been some evidences to show that these may in turn compromise their quality of life (QOL) [11, 12].

Majority of the studies on QOL in lymphoma survivors were limited to the western populations [13, 14]. A systematic review by Arden-Close et al. showed that the lymphoma survivors had a worse physical HRQOL than the normal 